\title{
El proceso de reforma del sector salud en Perú
}

\author{
Pedro Jesús Mendoza-Arana, ${ }^{1}$ Germán Rivera-Del Río, ${ }^{2}$ \\ César Gutiérrez-Villafuerte ${ }^{2}$ y César Sanabria-Montáñez ${ }^{2}$
}

Forma de citar: Mendoza-Arana PJ, Rivera-Del Río G, Gutiérrez-Villafuerte C, Sanabria-Montáñez C. El proceso de reforma del sector salud en Perú. Rev Panam Salud Publica. 2018;42:e74. https://doi.org/10.26633/ RPSP.2018.74

RESUMEN Objetivos. Caracterizar el proceso de la Reforma del Sector Salud (RSS) en Perú expresada públicamente en 2013, identificando los principales avances en su implementación y los desafios pendientes desde la perspectiva de los actores participantes.

Métodos. Se trata de un estudio de sistematización de la experiencia en el cual se realizaron entrevistas semiestructuradas a 21 informantes clave, incluyendo a tres exministros de salud, y empleando como marco temporal el decenio 2005-2015. Se analizaron bases de datos oficiales para comprobar las variaciones de los indicadores de salud.

Resultados. La propuesta se basa en la expansión del aseguramiento con predominio de un seguro público en salud bajo el modelo del pluralismo estructurado, con una clara separación entre las funciones de prestación, intermediación financiera, regulación y gobierno. Los principales avances de la RSS identificados son: haber trascendido el criterio de pobreza para el aseguramiento público, el refuerzo de la inversión física y de recursos humanos, el fortalecimiento de una superintendencia orientada a los derechos del usuario, y el del papel del Ministerio de Salud en la salud pública. Y los principales desafíos, la cobertura poblacional del aseguramiento no vinculada con la pobreza, la dotación de recursos humanos especializados y la reducción de gasto de bolsillo.

Conclusiones. La RSS en el decenio examinado es un proceso que se construye sobre avances de años precedentes al periodo analizado, que consolida en el país un modelo de aseguramiento encaminado a la cobertura poblacional universal sobre la base de un seguro público de salud, y que se expresa en un incremento demostrable del gasto público y de la cobertura, aunque sus avances se ven limitados principalmente en la dotación de recursos humanos especializados y en el gasto de bolsillo, que todavía es muy elevado.

Palabras clave Reforma de la atención de salud; sistemas de salud; seguro de salud; cobertura universal; financiación de la atención de la salud; Perú.

Los procesos de reforma en salud se llevan a cabo en todo el mundo buscando

\footnotetext{
Grupo de Investigación SYSTEMIC, Universidad Nacional Mayor de San Marcos, Lima, Perú. La correspondencia se debe dirigir a Pedro Mendoza-Arana, pmendozaa@unmsm.edu.pe

2 Universidad Nacional Mayor de San Marcos, Lima, Perú.
}

mejoras significativas en los sistemas de salud. Para los fines del presente estudio la reforma del sector salud (RSS) se define como el conjunto de cambios intencionados que tienen como objetivo principal producir cambios relevantes en más de uno de los siguientes dominios: la titularidad o el derecho a recibir tales prestaciones ("entitlement"), la variedad de prestaciones que ofrece el sector salud, la forma como se agencia el financiamiento de estas prestaciones, y las estructuras institucionales que componen el sector salud.

La bibliografía sobre este tema suele estar enfocada en los resultados o el

Este es un artículo de acceso abierto distribuido bajo los términos de la licencia Creative Commons Attribution-NonCommercial-NoDerivs 3.0 IGO, que permite su uso, distribución y reproducción en cualquier medio, siempre que el trabajo original se cite de la manera adecuada. No se permiten modificaciones a los artículos ni su uso comercial. Al reproducir un artículo no debe haber ningún indicio de que la OPS o el artículo avalan a una organización o un producto específico. El uso del logo de la OPS no está permitido. Esta leyenda debe conservarse, junto con la URL original del artículo. 
impacto sobre diversos indicadores epidemiológicos o de utilización de servicios (1-3) o expresa testimonios de parte (4), pero es escasa la sistematización de los procesos seguidos, que integre las perspectivas de diversos actores. En este artículo se aplica el método de la sistematización de la práctica con tres objetivos: 1) caracterizar el proceso seguido en la RSS en Perú formalizada en el período 2013-2015, 2) identificar los avances en su implementación, y 3) delinear los desafíos pendientes para la RSS.

\section{MATERIALES Y MÉTODOS}

Este estudio es una sistematización de la experiencia. De acuerdo con Oscar Jara (5), la sistematización es la interpretación crítica de las experiencias para descubrir o explicitar la lógica del proceso vivido en ellas y forma parte de los abordajes de la investigación cualitativa (6). Su enfoque central es la construcción del conocimiento en un proceso de diálogo con los propios actores que viven el proceso siguiendo lo propuesto por Sergio Martinic (7).

En los meses de mayo y junio de 2015 se realizaron 21 entrevistas semiestructuradas a informantes clave seleccionados por su vinculación directa con el proceso de la RSS. Estos informantes pertenecían al ente rector del proceso, el Ministerio de Salud (se incluyeron tres Exministros y un Exviceministro de salud) y a sus niveles operativos o eran representantes de los usuarios de los servicios de salud o investigadores o expertos de entidades académicas o representantes de agencias de cooperación. Las entrevistas las llevaron a cabo dos de los autores empleando una guía de entrevista semiestructurada con cuatro líneas: a) los actores de la RSS, b) las estrategias de la RSS, c) las intervenciones de la RSS, y d) los desafíos de la RSS.

Esta información se complementó con pruebas cuantitativas extraídas de encuestas de cobertura nacional en el período 2005-2015: la Encuesta Nacional de Demografía y Salud (ENDES), la Encuesta Nacional de Hogares (ENAHO) y la Encuesta Nacional de Satisfacción de Usuarios en Salud (ENSUSALUD). El objetivo de su utilización en el estudio fue contextualizar las referencias de los entrevistados e identificar variaciones en indicadores que pudieran reflejar efectos de las decisiones vinculadas con la RSS en sus componentes financiero, prestacional y de protección del derecho a la salud, así como en indicadores sanitarios seleccionados entre los relacionados con los Objetivos de Desarrollo del Milenio. Todas las aseveraciones incluidas en la sección de Resultados provienen de las entrevistas, excepto las referenciadas, porque proceden de encuestas o de estudios específicos. En cumplimiento de las normas éticas, todos los entrevistados fueron informados del objetivo de la entrevista y se mantienen en el anonimato.

\section{RESULTADOS}

\section{Caracterización de la RSS en Perú}

En el período de referencia que abarca este estudio, se fueron creando espacios de análisis en torno a las opciones y los modelos para la Reforma, que, siguiendo a uno de los entrevistados, se denominan "las Comunidades de la Reforma". Cuando se busca identificar el surgimiento de la propuesta de reforma actualmente en curso queda claro que no hay un modelo unitario de implementación. Uno de los entrevistados (Entrevistado 8) planteó este punto en los siguientes términos: "se van generando distintas "comunidades de reforma", y estas comunidades de reforma comienzan ya a tratar de colocar sus propuestas de reforma".

El consenso que surge de las entrevistas es que la propuesta de la RSS en Perú es una construcción social, configurada gradualmente en un proceso en el cual los entrevistados reconocen explícitamente algunos referentes, tanto nacionales, como, por ejemplo, el Acuerdo Nacional (8) y el Acuerdo de los Partidos Políticos en Salud (9), como internacionales.

Por parte de las agencias internacionales, en el $52^{\circ}$ Consejo Directivo de la Organización Panamericana de la Salud (OPS), celebrado en septiembre de 2013, los países de la Región, entre ellos Perú, reafirmaron su compromiso con la cobertura universal de salud (10).

Otro actor en este proceso ha sido la Agencia de los Estados Unidos para el Desarrollo Internacional (USAID), que ha participado en la RSS con diversos equipos de trabajo (11). Por su parte, el Banco Mundial identificó como sus principales contribuciones a la estrategia de trabajo en Perú el respaldo al Programa de Apoyo a la Reforma del Sector Salud II (PARSALUD II), a la descentralización de la prestación de servicios y a la mejora de la eficiencia del Seguro Integral de Salud
(SIS), así como a un seguro público generado en Perú dirigido inicialmente a las poblaciones en extrema pobreza (12-14).

Es importante subrayar la visibilidad que tiene el proceso en los medios de comunicación, incluidas las redes sociales virtuales, en los grupos de la sociedad civil, en entes gremiales - todos ellos con una posición crítica frente a la propuesta del Gobierno-, y en las organizaciones no gubernamentales.

Como primer hito visible del proceso desde el punto de vista de la sociedad debe mencionarse la Resolución Suprema 001-2013-SA, aprobada el 8 de enero de 2013, cinco meses después de la toma de posesión de la Ministra Midori de Habich (que ejerció el cargo del 28 de julio de 2012 al 5 de noviembre de 2014). Por medio de ella, encargó al Consejo Nacional de Salud formular y proponer acciones para la RSS al tiempo que invitó a participar a miembros de la sociedad civil, de Gobiernos Regionales, de organismos públicos y a diversos expertos y especialistas para darle un respaldo político amplio y explicitar la importancia de la descentralización y del papel de las regiones.

En general, el equipo vinculado con el Despacho Ministerial en 2013 se identifica como el formulador básico de las propuestas de la RSS del periodo en estudio. Desde la perspectiva de la sociedad civil, este proceso fue percibido, al menos por una parte de los actores, como un mero trámite para formalizar propuestas que se trabajaban en el propio Ministerio de Salud y luego se presentaban para su aprobación. Otros actores percibieron que el diálogo en realidad no fue tal, sino que se recibían opiniones que no se incorporaban a la propuesta.

Con sus avances e imperfecciones, todo ello culminó con el segundo hito: la publicación del documento Lineamientos y medidas de Reforma del Sector Salud (15), presentado por el Consejo Nacional de Salud en julio de 2013, que exhibe un conjunto de propuestas de mandato, fundamentadas y operacionalizadas con un horizonte temporal. Esta publicación precedió al mensaje presidencial ante el congreso, en el cual se pidió la delegación de facultades para legislar, que fue concedida, y aceleró el proceso. El tercer hito fue la promulgación de 23 dispositivos legales en diciembre de 2013, cumpliendo el plazo otorgado para ello por el Congreso. La RSS descrita en los documentos de 2013 se puede caracterizar 
como una reforma "Centrada en el Usuario". Este es un rasgo distintivo; de hecho, el Presidente de la República del Perú (que ocupó el cargo de julio de 2011 a julio de 2016) fue el único de los presidentes entre 2005 y 2015 que incluyó en su discurso pronunciado en 2012 ante el Congreso de la República una alusión a "elevar la satisfacción del usuario del actual $45 \%$ al $70 \%$ para 2016 ".

Con estos referentes el equipo ministerial construyó su propia aproximación y señaló que la RSS debería universalizar la protección en salud en el país como objetivo de política y, para ello, propuso un elemento transversal para conseguir su viabilidad y sostenibilidad: el fortalecimiento de la rectoría y de la gobernanza del sistema. La propuesta se basó en la expansión del aseguramiento en salud a partir de la ampliación del seguro público en salud de Perú (el Seguro Integral de Salud o SIS).

Otro componente nuclear de la propuesta fue la separación entre las funciones de prestación, intermediación financiera, regulación y gobierno. Esta separación y especialización se reflejó en modificaciones de la Ley de Organización y Funciones del Ministerio de Salud (MINSA), en la creación del Instituto de Gestión de Servicios de Salud, en el fortalecimiento del ente regulador, en la Superintendencia Nacional de Salud (SUSALUD), y en el fortalecimiento de las Instituciones Administradoras de Fondos del Aseguramiento en Salud (IAFAS). Los entrevistados del MINSA entendieron esta especialización como una fórmula mediante la cual el Ministerio se desprende de tareas operativas para concentrarse en la rectoría.

\section{Principales avances en el proceso de la RSS}

En el eje de protección poblacional, sobre la base de un sistema previsional, la principal estrategia fue transformar el SIS, de un programa de focalización del gasto, como fue inicialmente planteado y desarrollado, a un seguro público, trascendiendo el criterio de pobreza y convirtiéndolo en un criterio de vulnerabilidad, de manera que no solo los pobres fueran objeto de afiliación por parte del SIS.

De acuerdo con las ENAHO, entre 2005 y 2014 la cobertura poblacional de aseguramiento pasó de 36 a 69,7\% (de 39 a 66,7\% en zonas urbanas y de 30 a $78,7 \%$ en zonas rurales) y esta evolución tuvo una tendencia ascendente estadísticamente significativa.

Otro de los logros valorados fue el fortalecimiento del primer nivel de atención, tanto en lo referido al financiamiento, a través del pago capitado por parte del SIS, como en lo que atañe a los recursos humanos, mediante la expansión del Servicio Rural y Urbano Marginal de Salud (SERUMS). (El SERUMS es un programa instaurado en Perú en 1981 en el cual los profesionales de la salud recién titulados deben prestar un año de servicios remunerados en zonas rurales como requisito para aspirar a programas de especialización o a contratos con entidades públicas.)

Otro logro considerado relevante son las medidas para el incremento de los recursos humanos especializados. Dado el periodo de latencia de las medidas relacionadas con la formación de recursos humanos especializados, los logros en este periodo, más que reflejarse en una mayor dotación de especialistas, se reflejan en el incremento de recursos destinados a la formación especializada y en el establecimiento de normas para que el MINSA oriente mejor los procesos de formación, especialmente a través de las segundas especialidades de formación en servicio remuneradas, que se denominan "residentados".

Un avance claro en la capacidad de regulación del sistema fue la transformación de la Superintendencia Nacional para el Aseguramiento en Salud (SUNASA) en la Superintendencia Nacional de Salud (SUSALUD) merced al Decreto Legislativo 1158-2013, del 6 de diciembre de 2013. La SUSALUD asumió la capacidad de supervisar el cumplimiento de las normas sanitarias en entidades públicas y privadas y ha fortalecido notablemente el componente de promoción de los derechos del usuario.

Otro elemento fue el fortalecimiento del papel del MINSA en la salud pública con la creación de un nuevo Viceministerio con capacidad de intervención a nivel nacional. Hasta antes de la RSS el MINSA no podía intervenir en las regiones del país, porque las funciones de salud pública se habían transferido a los gobiernos subnacionales como parte de un proceso nacional de descentralización del Gobierno. El Decreto Legislativo 1156-2013, del 6 de diciembre de 2013, le permite al MINSA intervenir en una región previa declaratoria de emergencia sanitaria.
En lo referido al financiamiento, en los últimos diez años (2005-2014) en Perú se ha incrementado de manera sustantiva el gasto total en salud, pasando de un gasto per cápita de 435,00 a 784,00 soles (expresados en nuevos soles constantes de 2007) y de un gasto total de 10415,8 millones a 23625,7 millones de nuevos soles (16). Este incremento se explica esencialmente por un incremento del PIB nacional. Cuando se examina el gasto total en salud como porcentaje del PIB, este ha pasado de 4,4 a $5,2 \%$, pero con un comportamiento fluctuante; es decir, este porcentaje se ha mantenido hasta 2008 cuando superó la barrera de 5\%.

De manera similar, cuando se examina el comportamiento del gasto de bolsillo, se aprecia que ha ido aumentando hasta alcanzar un máximo de $42,51 \%$ en 2008 y que a partir de 2009, con el inicio de la política del Aseguramiento Universal en Salud, empezó a descender (17).

Este último es un indicador especialmente relevante, puesto que uno de los principios de la RSS es la protección financiera, que, a la luz del concepto internacional de financiamiento de los servicios de salud, se traduce en pasar de un esquema basado en el gasto en el punto de atención a un gasto previsto mediante la creación de los mecanismos de aseguramiento (10).

El gasto catastrófico en salud puede definirse como aquel gasto de bolsillo en salud que supera $10 \%$ de los ingresos de la familia (18). La protección de las familias en tal situación es uno de los elementos centrales de la propuesta de reforma desde el punto de vista de la protección financiera, y en lo referido a las familias en situación de pobreza y pobreza extrema, el instrumento utilizado es el SIS. Se esperaría entonces que la expansión del financiamiento ejecutado por el SIS se correlacionara con la reducción del gasto catastrófico en salud. La evidencia empírica disponible a este respecto es que las Cuentas Nacionales en Salud muestran que en el periodo 2004-2012 la probabilidad de gasto de un afiliado al SIS respecto a un no afiliado se redujo de 11,6 a 3,5 veces en el quintil 1, es decir, el de mayor pobreza. Sin embargo, entre 2006 y 2016 la pobreza en Perú se redujo de 49,1 a $20,7 \%$, y la pobreza extrema, de 13,8 a 3,8\%

\section{Principales desafíos}

Uno de los preceptos de la RSS es la separación de las funciones de prestación, 
intermediación financiera, regulación y gobierno. En cuanto a la prestación, con la descentralización política en curso en el país una parte importante de los establecimientos de salud son administrados por los gobiernos regionales; sin embargo, aquellos ubicados en la ciudad capital, Lima, todavía no han sido asumidos por el Gobierno Metropolitano y siguen bajo la responsabilidad del Ministerio de Salud. Perú no tiene un segundo nivel de atención con la fortaleza suficiente para contener la demanda de hospitales del nivel III, que debe articularse con la organización de redes y situando algunas especialidades fundamentales más cerca de la población.

Por otro lado, se hace más visible la necesidad de recursos humanos especializados crecientes, no solo de especialidades asistenciales, de las cuales hay una gran brecha, sino de las no asistenciales, como la epidemiología, la planificiación y la gestión, así como los recursos especializados para el aparato administrativo, como especialistas en informática sanitaria, equipos biomédicos, arquitectura sanitaria o inversiones en salud. En relación con lo primero, los entrevistados enfatizaron la necesidad de profesionalizar a los gestores, sobre todo a aquellos con menor experiencia, que son numerosos y la magnitud de los recursos que dependen de sus decisiones, enorme.

Por lo tanto, un tema que se va ir tornando más crítico en la medida en que se concrete la inversión en los establecimientos es la necesidad de disponer de recursos humanos especializados. Se han lanzado iniciativas, como el SERUMS de especialistas o la formación especializada de otros profesionales en las cuales se deberá seguir trabajando adecuadamente.

El aumento del gasto público en salud en la década precedente ha sido esencialmente el resultado de un incremento del porcentaje de gasto en salud respecto al presupuesto general de la República, de 2,3 en 1995 a $2,8 \%$ en 2012 en un contexto de incremento del PIB como resultado de una coyuntura internacional favorable (16). En los últimos años, hay señales congruentes de un enlentecimiento global, que tiene como resultado previsible una disminución del crecimiento del PIB. En este contexto, para poder mantener el crecimiento del gasto público, queda apelar al incremento de la participación relativa del gasto en salud hacia el promedio de la Región de las Américas de 4\%, por no mencionar la media de la OCDE, de 8\% (19).

\section{DISCUSIÓN}

Se ha descrito que los análisis de las políticas de salud son un área de investigación sumamente compleja no sólo por los métodos que emplea, sino por lo sensible que son sus hallazgos y por el inevitable posicionamiento de los actores (20). En este tipo de situaciones, la investigación cualitativa, que incluye la sistematización, es necesaria para comprender mejor los procesos estudiados $(6,21)$.

$\mathrm{Si}$ bien las reformas suelen mostrarse como construcciones en un momento determinado, el presente estudio sugiere que la propuesta actual de la RSS en Perú es una construcción social, configurada gradualmente, que reconoce aportaciones direccionales de 10 a 15 años antes $(3,4)$ e integra versiones actualizadas de mejoras introducidas en el sistema hace 20 años, como el concepto de seguro público introducido en Perú en 1997, si bien en aquella época no demostró haber aumentado la equidad del sistema (22).

Si este proceso se examina a la luz de los cuatro dominios planteados para la Reforma, en 2013 se ratificó el concepto de aseguramiento universal sobre la base del seguro público, es decir, se especificaron tanto elementos referidos a la titularidad como a la forma de financiamiento, se continuó mejorando el Plan Esencial para el Aseguramiento en Salud (PEAS), es decir, la variedad de prestaciones, y se rediseñaron las estructuras institucionales, con lo cual se cumplen los cuatro elementos inicialmente establecidos.

Por otro lado, aunque hay elementos que pueden rastrearse a 20 años vista, otros componentes, como el relativo a los derechos del paciente, son más recientes $\mathrm{y}$ han promovido un movimiento general en defensa de los derechos del consumidor (23).

El elemento pivotal de la reforma en la esfera financiera es el seguro público. Se ha demostrado que el aseguramiento público protege contra el gasto de bolsillo y el potencial gasto catastrófico derivado $(24,25)$. No obstante, en Perú el gasto de bolsillo parece mantenerse a pesar del incremento significativo del aseguramiento público, que se explica sobre todo por el gasto de las familias en medicamentos, lo cual sugiere que es necesaria una regulación fuerte de este mercado para que el aseguramiento surta el efecto deseado.

El aseguramiento ha hecho visible el déficit de recursos humanos especializados en el sistema de salud peruano, un problema que se pone de manifiesto en todos los países donde las propuestas de políticas de salud buscan cambios, tanto en América Latina (26) como en Europa (27). Esta brecha resulta más clara en razón de la mejora en las infraestructuras y de la demanda establecida por el propio PEAS, que indica con precisión en qué categoría de establecimientos se deben atender las enfermedades cubiertas por el plan y de lo cual se deriva con qué recurso humano se debe atender.

En estudios publicados en $2011(28,29)$ se estimó que para 2010 la brecha era de 13485 médicos especialistas, 5102 de ellos requeridos en el MINSA y 8383 en EsSalud. Esta diferencia es más dramática en algunas especialidades y tiende a incrementarse. Por añadidura, en una proyección prospectiva, se necesita estimar con la anticipación debida las necesidades de nuevas especialidades o subespecialidades que el sistema de salud va a necesitar. El sistema universitario tarda un mínimo de dos años para crear y poner en marcha nuevas especialidades. Esto, sumado al proceso formativo de tres a cinco años, supone una latencia mínima de cinco a ocho años para tener nuevos especialistas desde el momento en que se tome la decisión. Este es uno de los temas críticos en el plano de los recursos humanos, especialmente ante las pruebas disponibles de una emigración creciente de personal de salud $(30,31)$.

De todo lo expuesto se pueden extraer las siguientes conclusiones. Primero, en relación con la caracterización del proceso seguido en la formulación e implementación de la actual propuesta de RRS en Perú, se trata de un proceso de acumulación histórica de dos décadas, con adecuaciones relevantes enfocadas hacia los derechos del ciudadano en los últimos siete años, que se han articulado de manera coherente en la propuesta de 2013. Segundo, los avances identificados en dicha implementación, según lo identifican los propios actores, son a) 
haber trascendido el criterio de pobreza para el aseguramiento público, b) el fortalecimiento de la rectoría a través de la creación de una Superintendencia orientada a los derechos del usuario y del Viceministerio de salud pública, y c) el refuerzo de la inversión física y de recursos humanos. Tercero, los principales desafíos pendientes de la RSS, según los identifican sus propios actores, son la brecha de cobertura poblacional del

1. Sandeep R, Jones P, Shanthanna Darsha Damarell R, Wakerman J. A Systematic Review of the Impact of Healthcare Reforms on Access to Emergency Department and Elective Surgery Services: 1994-2014. Int J Health Serv. 2018;48(1):81-105.

2. Bagheri Lankaran K, Khankeh HR, Zarei N, Fararouei M, Saboori Z, Joulaei H. Toward Equity under Health System Reform; A Systematic Review. Shiraz E Med J. 2017; 18(11):e57724.

3. Carter R, Riverin B, Levesque JF, Gariepy G, Quesnel-Vallee A. The impact of primary care reform on health system performance in Canada: a systematic review. BMC Health Serv Res. 2016;16:324.

4. Velásquez A, Suárez D, Nepo-Linares E. Reforma del sector salud en el Perú: derecho, gobernanza, cobertura universal y respuesta contra riesgos sanitarios. Rev Peru Med Exp Salud Publica. 2016;33(3):546-55.

5. Holliday OJ. La Sistematización de Experiencias: Teoría y Práctica para otros mundos posibles. Lima: Intermon Oxfam; 2012.

6. Lenise Do Prado M, de Souza ML, Monticelli M, Cometto MC, Gómez PF. Investigación Cualitativa en Enfermería. Metodología y Didáctica. Washington, DC: Organización Panamericana de la Salud; 2013.

7. Martinic S. El objeto de la sistematización y sus relaciones con la evaluación y la investigación. Ponencia presentada al Seminario Latinoamericano Sistematización de prácticas de animación sociocultural y participación ciudadana en América Latina, Medellín, 12-14 de agosto de 1998. Medellín: Fundación Universitaria Luis AmigóCEAAL; 1998.

8. Disponible en: http:/ /acuerdonacional.pe/ politicas-de-estado-del-acuerdo-nacional/ Acceso el 7 de abril de 2018.

9. Disponible en: http:/ / www.unfpa.org.pe/ programa/acuerdoensalud.pdf Acceso el 7 de abril de 2018.

10. Organización Panamericana de la Salud. Resolución CD53.R14. Estrategia para el acceso universal a la salud y la cobertura universal de salud. Washington, DC: OPS; 2014.

11. de Habich M, Madueño M, Sobrevilla A. El Plan Esencial de Aseguramiento en Salud: fundamentos técnicos y metodológicos. aseguramiento no vinculada con la pobreza, las brechas notables y crecientes en la dotación de recursos humanos especializados, y el escaso impacto de la RSS en la reducción del gasto de bolsillo.

Financiación. El presente artículo resume el Informe Final de una consultoría para la Representación en Perú de la Organización Panamericana de la Salud,

\section{REFERENCIAS}

Bethesda, MD: Health Systems 20/20 project, Abt Associates Inc; 2009.

12. United States Agency for International Development, Peru. USAID/Peru Country Development Cooperation Strategy. Lima: USAID/Peru; 2012.

13. Interamerican Development Bank, Peru. Peru: IDB country strategy with Peru 2012-2016. Washington, DC; IDB/Peru; 2012.

14. World Bank. Alianza Estratégica con el País para la República del Perú. Ejercicios 2012-2016. Washington, DC: World Bank; 2012.

15. Consejo Nacional de Salud. Lineamientos y medidas de reforma del sector salud. Lima: Ministerio de Salud; 2013.

16. Ministerio de Salud. Cuentas Nacionales de Salud 1995-2012. Lima: Ministerio de Salud, Organización Panamericana de la Salud; 2015.

17. Velásquez A. Salud en el Perú: Hacia la cobertura universal y una respuesta efectiva frente a riesgos sanitarios. Rev Peru Med Exp Salud Publica. 2016;33(3): 397-8.

18. Raban MZ, Dandona R, Dandona L. Variations in catastrophic health expenditure estimates from household surveys in India. Bull WHO. 2013;91:726-735.

19. Disponible en: http://blogs.iadb.org/sa$\mathrm{lud} / 2014 / 06 / 03 /$ el-gasto-de-salud-enamerica-latina-engorda-pero-su-capacidad-financiera-no-gana-tantos-kilos / Acceso el 7 de abril de 2018.

20. Walt G, Shiffman J, Schneider H, Murray SF, Brugha R, Gilson L. 'Doing' health policy analysis: methodological and conceptual reflections and challenges. Health Pol Plann: 2008;23(5):308-17.

21. Do Prado ML, de Souza ML, Carraro TL. Investigación Cualitativa en Enfermería. Contexto y Bases Conceptuales. Washington, DC: Organización Panamericana de la Salud; 2013.

22. Jaramillo M, Parodi S. El Seguro Escolar Gratuito y el Seguro Materno Infantil: Análisis de su incidencia e impacto sobre el acceso a los servicios de salud y sobre la equidad en el acceso. Lima; GRADE; 2004. (Documento de trabajo 46.)

23. Gobierno Peruano. Ley 29571. Año 2010.

24. Aryeetey GC, Westeneng J, Spaan E, JehuAppiah C, Agyepong IA, Baltussen R. Can financiado a través del contrato PE CNT 1500046.001.

Conflictos de interés. Los autores declaran no tener conflictos de interés.

Declaración. Las opiniones expresadas en este manuscrito son responsabilidad de los autores y no reflejan necesariamente los criterios ni la política de la RPSP/ PAJPH y/o de la OPS. health insurance protect against out-ofpocket and catastrophic expenditures and also support poverty reduction? Evidence from Ghana's National Health Insurance Scheme. Int J Equity Health. 2016;15(116): 1-11.

25. Aji B, De Allegri M, Souares A, Sauerborn R. The Impact of Health Insurance Programs on Out-of-Pocket Expenditures in Indonesia: An Increase or a Decrease? Int J Environ Res Public Health. 2013;10(7): 2995-3013.

26. Alcalde-Rabanal JE, Nigenda G, Bärnighausen T, Velasco-Mondragón HE, Darney BG. The gap in human resources to deliver the guaranteed package of prevention and health promotion services at urban and rural primary care facilities in Mexico. Human Res Health. 2017;15(49): $1-11$.

27. Betliy OV, Kuziakiv OV, Onishchenko KV. The evaluation of health care system in Ukraine in the context of structural and quality-enhancing reforms. Moscow: Economics Education and Research Consortium; 2007.

28. Zevallos L, Pastor R, Moscoso B. Oferta y demanda de médicos especialistas en los establecimientos de salud del Ministerio de Salud: brechas a nivel nacional, por regiones y tipo de especialidad. Rev Peru Med Exp Salud Publica. 2011;28(2):177-85.

29. Balabarca P. Necesidad de Médicos Especialistas en EsSalud, Sanidades de las Fuerzas Armadas y PNP, año 2010. Lima: Ministerio de Salud; 2011.

30. Núñez M. Migración de Recursos Humanos en Salud. Lima: Organización Panamericana de la Salud; 2006.

31. Padilla M, Dreesch N. Migración Calificada en Salud, impacto financiero, reconocimiento de títulos: Retos y perspectivas en los países de la región andina. Lima: Organización Panamericana de la Salud; 2013.

Manuscrito recibido el 9 de septiembre de 2017. Aceptado para publicación, tras revisión, el 7 de febrero de 2018 . 
ABSTRACT Objective. To characterize the process of health sector reform (HSR) in Peru (launched publicly in 2013), identifying the principal advances in its implementation and the pending challenges from the perspective of the participating actors.

The process of health sector reform in Peru

Keywords Health care reform; health systems; insurance, health; universal coverage; healthcare

Methods. This study systematizes experiences through semi-structured interviews conducted with 21 key informants, including three ex-ministers of health, using the decade 2005-2015 as the time frame. Official databases were analyzed to verify variations in health indicators.

Results. The proposed reform was based on expanding insurance coverage (predominantly public health insurance), following the structured pluralism model, with clear separation between the functions of delivery, financing, regulation, and governance. The main progress in HSR identified by this study involves: having transcended the poverty criterion for public insurance, strengthening investments in infrastructure and human resources, strengthening the National Health Authority with a focus on the rights of users, and reinforcing the public health role of the Ministry of Health. The main challenges involve providing non-poverty-related insurance coverage for the population, having sufficient specialized human resources, and reducing out-of-pocket expenditure.

Conclusions. In the 10 years under analysis, HSR is a process that builds on the progress made in prior years; a process that consolidates an insurance model aimed at universal coverage based on public health insurance and that has led to a demonstrable increase in public spending and population coverage. However, progress has been limited mainly due to insufficient provision of specialized human resources and out-of-pocket expenditure, which remains very high.

$$
\text { financing; Peru. }
$$

RESUMO

\section{Processo de reforma da saúde no Peru}

Palavras-chave 